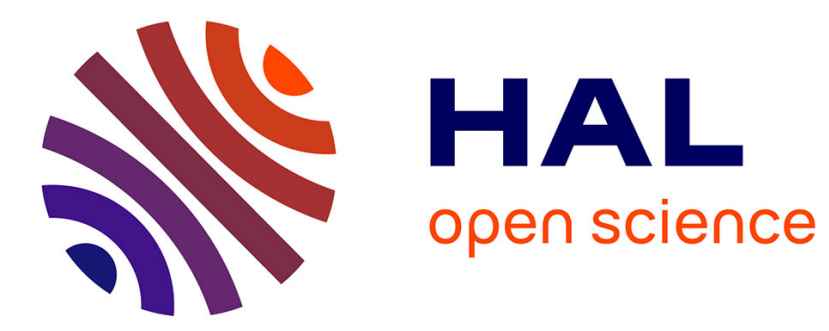

\title{
Couches minces supraconductrices à base D'YBa2Cu3O(7-x) par pulvérisation cathodique multicible
}

E. Bouteloup, B. Mercey, G. Poullain, Thierry Brousse, H. Murray, B. Raveau

\section{- To cite this version:}

E. Bouteloup, B. Mercey, G. Poullain, Thierry Brousse, H. Murray, et al.. Couches minces supraconductrices à base D'YBa2Cu3O $(7-\mathrm{x})$ par pulvérisation cathodique multicible. Revue de Physique Appliquée, 1990, 25 (2), pp.183-187. 10.1051/rphysap:01990002502018300 . jpa-00246177

\section{HAL Id: jpa-00246177 \\ https://hal.science/jpa-00246177}

Submitted on 1 Jan 1990

HAL is a multi-disciplinary open access archive for the deposit and dissemination of scientific research documents, whether they are published or not. The documents may come from teaching and research institutions in France or abroad, or from public or private research centers.
L'archive ouverte pluridisciplinaire HAL, est destinée au dépôt et à la diffusion de documents scientifiques de niveau recherche, publiés ou non, émanant des établissements d'enseignement et de recherche français ou étrangers, des laboratoires publics ou privés. 


\title{
Couches minces supraconductrices à base $\mathrm{D}^{\prime} \mathrm{YBa}_{2} \mathrm{Cu}_{3} \mathrm{O}_{(7-x)}$ par pulvérisation cathodique multicible
}

\author{
E. Bouteloup, B. Mercey, G. Poullain, T. Brousse, H. Murray et B. Raveau \\ CRISMAT ISMRa, Bd. du Mal. Juin, 14032 Caen Cedex, France
}

(Reçu le 31 juillet 1989, révisé le 29 septembre 1989, accepté le 19 octobre 1989)

\begin{abstract}
Résumé. - Cet article présente une technique inédite d'élaboration de couches minces supraconductrices à base d'Y $\mathrm{Ba}_{2} \mathrm{Cu}_{3} \mathrm{O}_{(7-x)}$. Le dispositif de pulvérisation cathodique multicible décrit permet, de par son architecture, la production simultanée et reproductible d'un nombre important de dépôts possédant une composition métallique très proche de $\mathrm{Y}_{17 \%} \mathrm{Ba}_{33} \mathrm{Cu}_{50 \%}$. Des films supraconducteurs $(R=0)$ à $80 \mathrm{~K}$ sur substrats de zircone polycristalline sont finalement obtenus après un recuit à haute température.
\end{abstract}

\begin{abstract}
This paper reports a new technique to prepare superconducting $\mathrm{YBa}_{2} \mathrm{Cu}_{3} \mathrm{O}_{(7-x)}$ thin films. The multitarget sputtering apparatus described below allows the simultaneous and reproducible production of numerous films with a metallic composition close to $\mathrm{Y}_{17 \%} \mathrm{Ba}_{33 \%} \mathrm{Cu}_{50 \%}$. Superconducting films $(R=0)$ at $80 \mathrm{~K}$ have been produced on polycrystalline zirconia substrates after a high temperature annealing.
\end{abstract}

\section{Introduction.}

Depuis le début 1987 , les nouveaux oxydes supraconducteurs à haute $T_{\mathrm{c}}$ ont fait l'objet de nombreuses recherches. Ils sont maintenant présents sous de multiples formes : céramiques, fils, couches épaisses et couches minces.

Etant donné l'enjeu économique représenté, il est important de trouver dès maintenant un compromis entre les performances atteintes et le coût des équipements employés afin de faciliter un éventuel transfert technologique vers l'industrie. Dans cette perspective les méthodes de pulvérisation cathodique présentent l'avantage de fournir des dépôts de qualité reproductible et d'obtention aisée.

Le dépôt à partir d'une seule cible, a priori le plus séduisant, est difficile à maîtriser en raison des écarts de composition observés entre films déposés et cibles utilisées. Ainsi, pour obtenir un film de composition donnée, on doit ajuster la composition de la cible ou partant d'une cible stœchiométrique modifier, de manière importante par rapport à un processus classique, la mise en œuvre du dépôt (substrat décentré, pression très élevée, etc.) [1].

Une autre méthode consiste, grâce à une disposition géométrique adaptée, à pulvériser séparément et dans des proportions ajustables les différents constituants du matériau supraconducteur. Cette technique «multicible », souvent rencontrée dans le domaine de l'évaporation par canons à électrons sous ultra-vide [2], n'a été jusqu'à présent adaptée à la pulvérisation cathodique que par quelques laboratoires [3]. C'est cette voie que nous avons pourtant décidé d'emprunter, choisissant la phase $\mathrm{YBa}_{2} \mathrm{Cu}_{3} \mathrm{O}_{(7-x)}$ comme matériau supraconducteur et trois cibles à base d'yttrium, de baryum et de cuivre comme sources des constituants.

\section{Techniques expérimentales.}

Dans une première série de manipulations, nous avons tenté de réaliser, en un même point $P$, la copulvérisation simultanée des trois constituants dans les proportions $1 / 2 / 3$.

Nous avons, pour ce faire, employé un groupe de pulvérisation ALCATEL SCM 450 sensiblement modifié.

La structure du module de pulvérisation constitué de trois cibles de diamètre $50 \mathrm{~mm}$ présente un axe de symétrie ternaire. Chacun des trois porte-cibles forme un angle de 25 degrés avec l'axe de symétrie du système (Fig. 1). Cette valeur résulte d'un compromis entre la volonté de provoquer la convergence des dépôts (point $\mathrm{P}$ ) aussi près que possible 


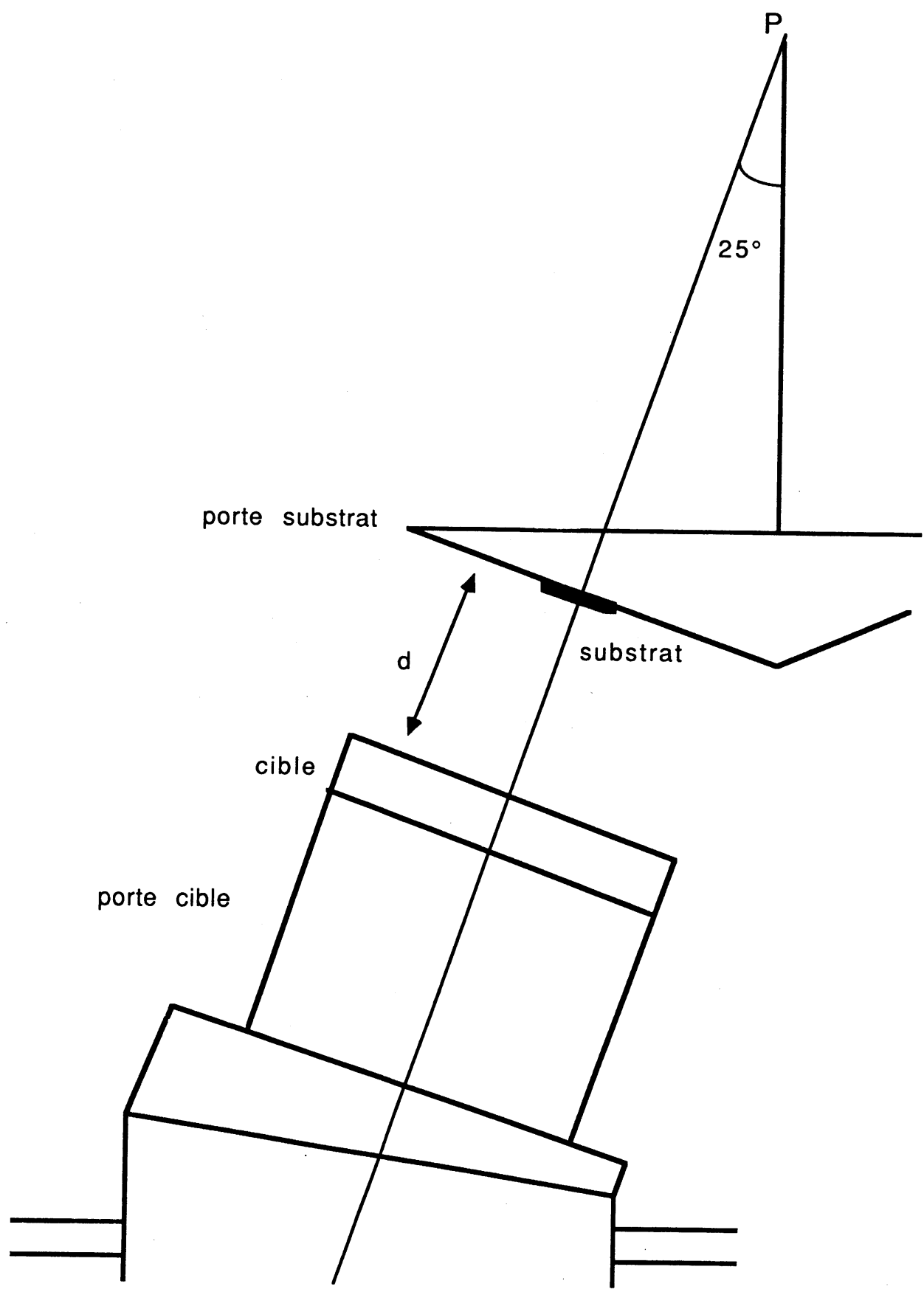

Fig. 1. - Schéma réduit du dispositif de pulvérisation $(d=20 \mathrm{~mm})$.

[Schematic diagram of the sputtering apparatus $(d=20 \mathrm{~mm})$.]

des cibles et la nécessité de limiter les interférences entre plasmas.

Concernant les éléments $\mathrm{Y}$ et $\mathrm{Cu}$, notre choix s'est immédiatement porté sur les cibles métalliques de composition $\mathrm{Y}$ et $\mathrm{Cu}$. Celles-ci, stables et conductrices présentent l'avantage de pouvoir être pulvérisées sous tension continue.

Pour le baryum, le choix de la cible s'avère beaucoup plus délicat. Des essais préliminaires effectués avec une cible de $\mathrm{BaO}$ ont mis en évidence une rapide détérioration de cette dernière. $\mathrm{Le} \mathrm{BaO}$ ainsi que le baryum métallique sont en effet instables au contact de l'air. Nous nous sommes orientés, comme l'avaient fait d'autres équipes [4-6], vers une cible plus stable constituée de fluorure de baryum $\mathrm{BaF}_{2}$.

L'ensemble ainsi constitué comporte :

- une cible d'yttrium reliée à une alimentation continue $0 / 50 \mathrm{~mA}$ sous $0 / 2000$ volts ;

- une cible de cuivre reliée à une seconde alimentation continue identique à la première ; 
- une cible de $\mathrm{BaF}_{2}$ reliée à un générateur $\mathrm{HF}$ $(13,56 \mathrm{MHz})$ d'une puissance maximale de 300 watts. Les cibles diélectriques supportant mal l'échauffement de leur surface, nous avons fixé une limite supérieure de 70 watts à la puissance HF appliquée, valeur réalisant un bon équilibre entre rendement de pulvérisation du fluorure de baryum et contraintes thermiques subies par la cible.

Après obtention d'un vide limite inférieure à $10^{-7}$ mbars, le plasma est amorcé en atmosphère d'argon $\left(P=2 \times 10^{-2}\right.$ mbars $)$ par la mise sous tension HF de la cible isolante. On procède ensuite par l'intermédiaire des alimentations continues à la polarisation des deux dernières cibles.

Cette technique de dépôt par co-pulvérisation simultanée a été abandonnée assez rapidement car nous nous sommes heurtés au problème fondamental suivant : dans les zones de l'espace où l'on pourrait en théorie recueillir la composition $1 / 2 / 3$, la répulsion des plasmas est telle que le taux de dépôt y est nul. Nous avons même observé dans certains cas une attaque ionique du substrat. Cette constatation annihile tout espoir d'obtenir des dépôts de composition $1 / 2 / 3$ au point $P$ défini précédemment.

Le module existant a donc été modifié afin de recueillir de façon séquentielle et non plus simultanée les éléments nécessaires à l'obtention d'un film de composition 1/2/3.

Dans ce but, nous avons réalisé un porte-substrat tournant pyramidal à base hexagonale. D'une surface équivalente à celle de la résistance chauffante du système initial, cette base est placée en regard de cette dernière. L'angle au sommet de la pyramide vaut 130 degrés. Chacune des faces de la pyramide pénètre de ce fait séquentiellement et parallèlement aux cibles chacun des trois plasmas (Fig. 1). Cette pyramide est en cuivre pour faciliter les transferts thermiques et éviter toute possibilité de contamination des dépôts par rétropulvérisation.

Compte tenu de la rotation, le chauffage ne peut se faire que par rayonnement mais la température maximale enregistrée a néanmoins pu être portée à $650^{\circ} \mathrm{C}$ en limitant la distance entre la résistance de chauffage et la pyramide.

La vitesse de rotation choisie égale à 1 tour/minute nous semble suffisamment rapide pour réaliser, à l'échelle atomique, un mélange intime des différents constituants.

Dans la configuration adoptée, la composition est proportionnelle aux rendements de pulvérisation des trois cibles [7].

Nous avions, dans un premier temps, envisagé un étalonnage préalable du taux de dépôt de chacune des trois cibles utilisée seule mais les trois rendements de pulvérisation étant interdépendants nous avons adopté la démarche suivante :

— pour des raisons déjà explicitées, la puissance
HF appliquée au fluorure de baryum a été fixée à 70 watts ;

- la tension continue imposée à l'yttrium a ensuite été ajustée de manière à obtenir un rapport $\mathrm{Ba} / \mathrm{Y}$ égal à 2 ;

- la tension continue imposée au cuivre a finalement été ajustée de manière à obtenir un rapport $\mathrm{Cu} / \mathrm{Ba}$ égal à 1,5.

Cette procédure a permis, après seulement quelques manipulations d'ajustage, d'obtenir un dépôt de composition très proche de $1 / 2 / 3$, le processus étant ensuite tout à fait reproductible.

Les dépôts sont effectués à froid en raison de la présence du fluor qui rend obligatoire un recuit ultérieur à haute température. Après obtention du dépôt, le recuit est immédiatement effectué selon le processus classique suivant.

— Montée lente à la vitesse de $150^{\circ} \mathrm{C} / \mathrm{h}$.

- Palier de 30 minutes à $900{ }^{\circ} \mathrm{C}$.

- Descente lente à la vitesse de $150^{\circ} \mathrm{C} / \mathrm{h}$.

Les deux premières étapes sont effectuées sous atmosphère d'oxygène saturé de vapeur d'eau afin d'effectuer l'hydrolyse des fluorures; La dernière, sous oxygène seul.

Les substrats utilisés sont constitués de zircone polycristalline stabilisée à l'yttrium.

\section{Résultats expérimentaux.}

3.1 CARACTÉRISTIQUES DES DÉPÓtS. - Le traitement (logiciel d'analyse TRACOR) du spectre $X$ recueilli en dispersion d'énergie par une microsonde de silicium associée à un microscope à balayage JEOL, permet d'accéder à la composition des dépôts dès leur sortie de groupe. Cette technique nous a permis d'estimer à environ $2 \mathrm{~cm}^{2}$ autour de leur centre géométrique le domaine d'homogénéité en composition de chaque face et de vérifier que chacune d'entre elles délivre un dépôt identique à celui de ses voisines. Chaque manipulation offre donc la possibilité de recueillir simultanément six dépôts identiques. Les échantillons sont noirs, brillants et isolants en sortie de groupe. Nous observons une rapide évolution des dépôts dès leur mise en contact avec l'air ambiant et ce d'une manière d'autant plus intense que leur teneur en baryum est plus importante. Ce phénomène peut sans doute être attribué à un début de réaction de carbonatation du baryum.

\subsection{CARACTÉRISTIQUES DES FILMS OBTENUS APRÈS} RECUIT. - Le logiciel d'analyse a permis de vérifier que la composition des dépôts effectués sur zircone n'évolue que très faiblement lors du recuit. L'analyse qualitative effectuée avec la micro-sonde et une fenêtre ultra-mince ne nous a pas permis de déceler de traces de fluorures dans les films recuits. Il 
semble donc que la substitution oxygène/fluor soit quasimént totale à $900^{\circ} \mathrm{C}$, résultat déjà mis en évidence par Chan et al. [8]. Les photographies prises au microscope à balayage montrent une microstructure en forme de bâtonnets très souvent observée dans le cas de la phase $\mathrm{YBa}_{2} \mathrm{Cu}_{3} \mathrm{O}_{(7-x)}$ mais non pas d'orientation préférentielle ceci s'expliquant en grande partie par le caractère polycristallin des substrats (Fig. 2). Des observations effectuées en coupe ont, quant à elles, permi d'estimer à un micron l'épaisseur obtenue pour un temps de dépôt de cinq heures. Les valeurs enregistrées à température ambiante pour les résistances sont comprises entre 2 et $10 \mathrm{ohms}$, soient des résistivités de l'ordre du mohm.cm. Le tracé $R=f(T)$ (Fig. 3) obtenu par la méthode des quatre points à partir de $300 \mathrm{~K}$ montre un comportement métallique avec une transition résistive rapide débutant aux environs de $90 \mathrm{~K}$ et s'achevant à $80 \mathrm{~K}$ (critère $: R / R(273 \mathrm{~K})$ inférieur à $10^{-4}$ ). Le courant critique relevé à $77 \mathrm{~K}$ est de l'ordre de $100 \mathrm{~A} / \mathrm{cm}^{2}$. Les résultats obtenus en mesure magnétique (SQUID) sur des couches minces de $5 \times 3 \mathrm{~mm}^{2}$ et dans des conditions difficiles (faible signal) confirment un début de transition vers $85 \mathrm{~K}$ (Fig. 4).

\section{Conclusion.}

Le dispositif présenté dans cette publication permet la production simultanée et reproductible d'un nombre important de films supraconducteurs. L'architecture porte-cibles/porte-substrats adoptée conduit à une vitesse de dépôt (env. $3 \mathrm{~nm} /$ minute) supérieure

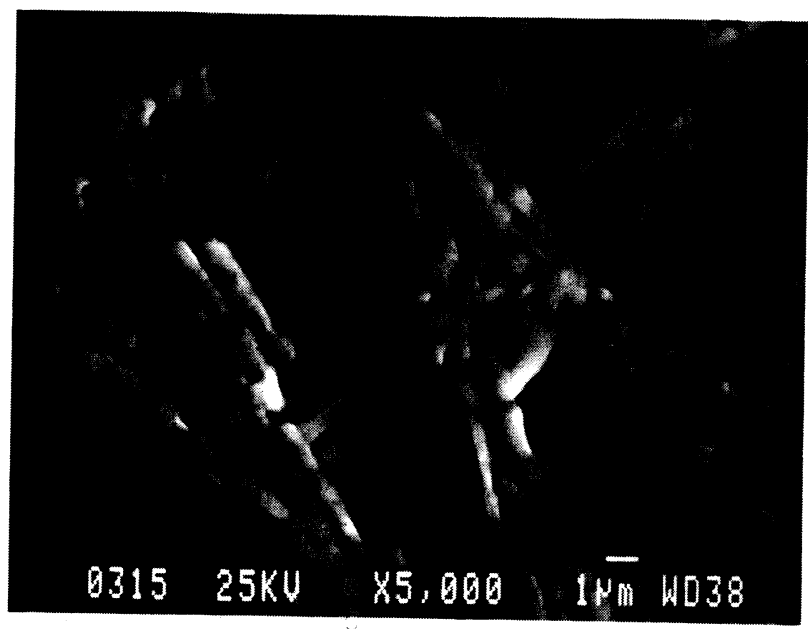

Fig. 2. - Cliché de microscopie électronique à balayage d'une couche après recuit sous oxygène.

[Scanning electronic micrograph of a thin film after annealing under oxygen.]

à celle qu'on obtiendrait avec des porte-cibles parallèles et un porte-substrats plan; le pourcentage du temps passé par chacun des substrats en face des cibles est en effet optimisé. Cependant, l'obtention de couches minces aux caractéristiques cristallographiques et microstructurales plus satisfaisantes nécessite une croissance in situ, sur substrat chauffé, en présence d'oxygène ainsi que la suppression de tout recuit à haute température après le dépôt $[9$,

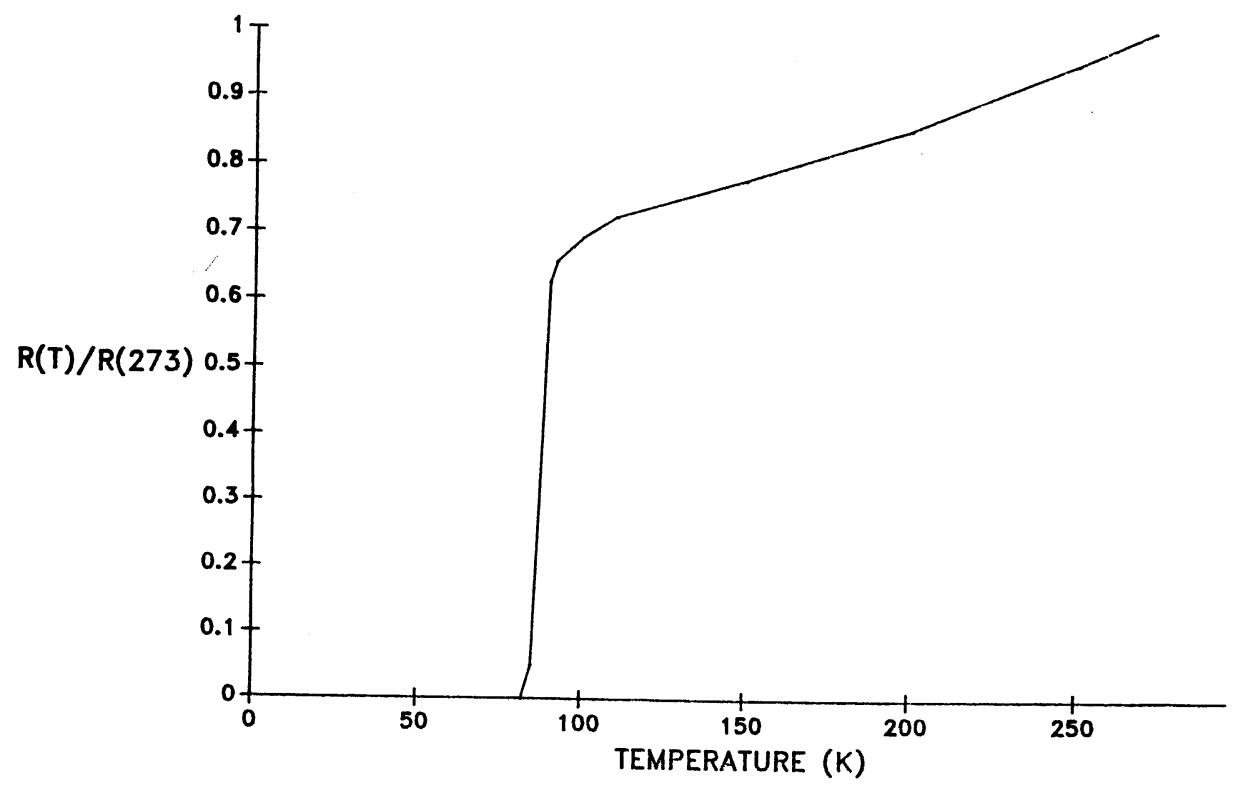

Fig. 3. - Courbe Résistance normalisée/Température relevée avec un courant de mesure de 50 microampères.

[Normalized resistance/Temperature curve taken with a measuring current of 50 microamperes.] 


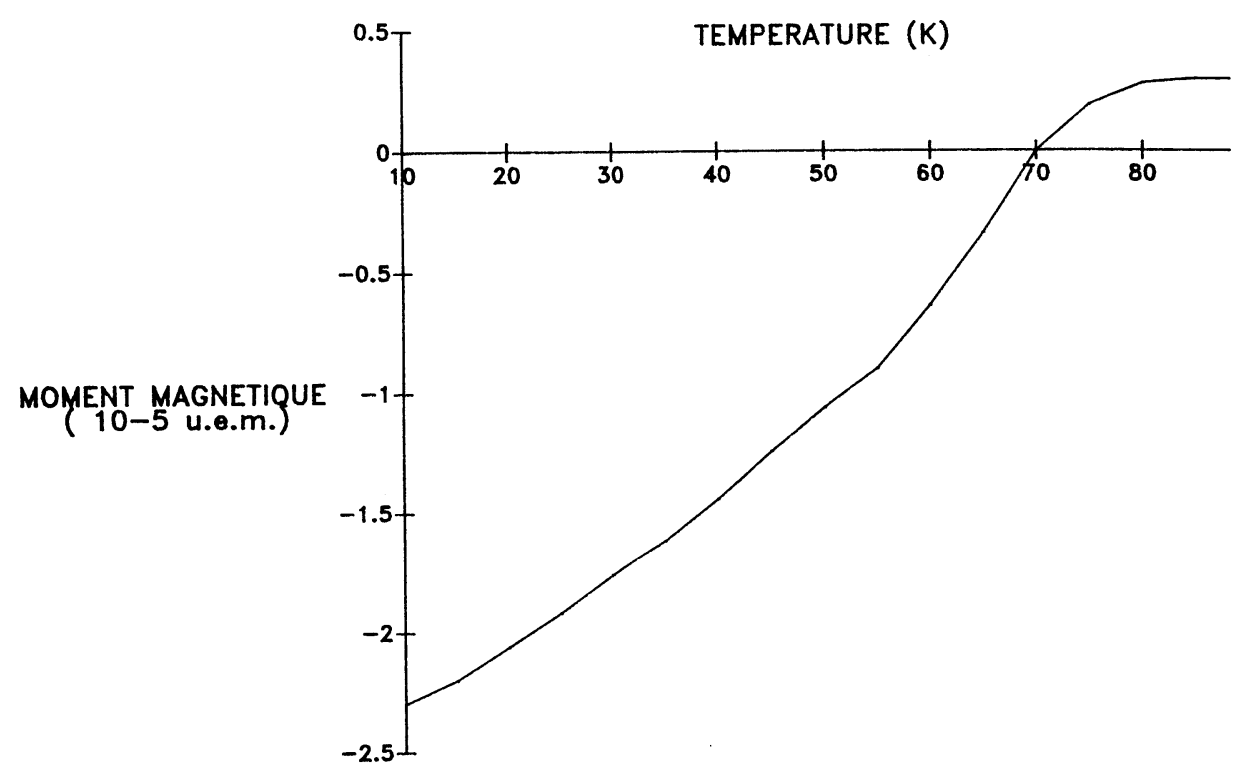

Fig. 4. - Courbe Moment magnétique/Température obtenue sous un champ de 200 gauss.

[Magnetic momentum/Temperature curve in a field of 200 gauss.]

10]. Le fluorure de baryum n'étant pas adapté au suivi d'une telle procédure, la cible de $\mathrm{BaF}_{2}$ a récemment été remplacée par une cible à base d'un oxyde mixte de baryum et de cuivre $: \mathrm{BaCuO}_{2}$. Les résultats préliminaires sont très prometteurs puisque nous avons déjà obtenu, sur zircone polycristalline et en conservant un mode opératoire classique (dépôt à froid, recuit ex situ), des couches minces supraconductrices d'une qualité comparable à celle obtenue avec la cible de $\mathrm{BaF}_{2}$.

\section{Bibliographie}

[1] Teradan N. et al., Jpn J. Appl. Phys. Lett. 27 (1988) L639.

[2] Miura T., Terashima Y., Sagoi M. and Kubo K., Jpn J. Appl. Phys. Lett. 27 (1988) L1260.

[3] Char K., Kent A. D., Kapitulnik A., Beasley M. R. and Geballe T. H., Appl. Phys. Lett. 51 (1987) 1370.

[4] Liou S. H. et al., Appl. Phys. Lett. 52 (1988) 1735. [5] Mogro-Campero A., Turner L. G., Hall E. L. and Burell M. C., Appl. Phys. Lett. 52 (1988) 2068.
[6] Moriwaki K., Enomoto Y., Kubo S. and Murakami T., Jpn J. Appl. Phys. Lett. 27 (1988) L2075.

[7] MAISSEL and Glang, Handbook of Thin Film Technology, 4-17 (1970).

[8] Chan S. W. et al., Appl. Phys. Lett. 53 (1988) 1443.

[9] Adachi H. et al., Appl. Phys. Lett. 51 (1987) 2263.

[10] TомiтA M. et al., Jpn J. Appl. Phys. Lett. 27 (1988) L636. 